\title{
Disseminated Intravascular Coagulation in Lung Cancers of Epithelial Origin
}

\author{
Debjit Chattopadhyay ${ }^{1}$, Shouvanik Adhya ${ }^{2}$, Soumyajyoti Bandyopadhyay ${ }^{2}$, \\ Somanjana Ghosh ${ }^{3}$, Subarna Goswami ${ }^{4}$, Arista Lahiri ${ }^{5}$ \\ ${ }^{I}$ (Department of Pathology, College of Medicine \& JNM Hospital, WBUHS, Kalyani, West Bengal, India) \\ ${ }^{2}$ (Department of Forensic Medicine, College of Medicine \& JNM Hospital, WBUHS, Kalyani, West Bengal, \\ India) \\ ${ }^{3}$ (Department of Microbiology, Midnapur Medical College, Midnapur, West Bengal, India) \\ ${ }^{4}$ (ACMOH, Swasthya Bhawan, Saltlake, Kolkata, West Bengal, India) \\ ${ }^{5}$ (Internee, R.G. Kar Medical College \& Hospital, Kolkata, West Bengal, India)
}

\begin{abstract}
Lung cancer is one of the commonest cancers in the world as well as in our country like India where smoking is that much prevalent. Epithelial lung cancers are common in smokers and one of its commonest manifestations is DIC especially chronic DIC. Our aim is to find out the prevalence of DIC in epithelial lung cancers so that DIC related catastrophes can be minimized and better treatment protocols can be formulated. Complete hemogram, PT, APTT, platelet count, and FDP D 'Dimer assay is done on 35 patients with diagnosed lung cancers of epithelial origin and on 35 healthy controls to prove the accuracy. It is found that 19 (54.28\%) cases show raised FDP D Dimer and other findings like normal PT \& APTT in most of the cases confirms chronic DIC. It can be concluded that chronic DIC is a common event in epithelial lung cancers and proper recognition of this event may further improve treatment protocols.
\end{abstract}

Keywords: APTT, DIC, Epithelial lung cancers, FDP D Dimer, Platelet count, PT

\section{Introduction}

Haemostatic defects in malignancy may be in haemostasis itself or fibrinolytic systems or in platelets. Disseminated intravascular coagulation (DIC) is one of the commonest haemostatic alterations observed in cancer patients.

DIC may be acute or chronic. Lung cancer of epithelial origin is a common cause of chronic DIC. Pathway remaining the same acute DIC occurs in an extrusive manner and chronic is slow and much less explosive. Lung cancer of epithelial origin is associated with chronic DIC.

Studies are performed with the aim of preventing any DIC-related catastrophe in the course of epithelial lung cancers andlor in their treatment. Mersky C, Johnson A.J, Kleiner P.J. ${ }^{[1]}$ evaluated the defibrination syndrome and noted the event of DIC is common in cancer and also in lung cancers.

As because smoking is very much preponderant in our country, lung cancer is increasing by leaps and bounds. It is most common cancer in male and third most cancer in females. As because our countrymen are less health conscious they usually present in later stages of the disease with much more associated complication and side effects making the treatment procedures all the more difficult.

So we have to throw light on these side effects also so that a better protocol can be formulated to alleviate symptoms as well as to help curative therapy. Disseminated Intravascular Coagulation or DIC of chronic nature is one of such common and much disturbing complication of epithelial origin carcinomas of lung. For this reason meticulous study and investigations are necessary for this kind of DIC.

As we all know this is an acquired type of DIC where tissue factors play the role of match-stick to activate the extrinsic pathway here vesicles and thromboplastin like cancer related products activates the extrinsic pathway initiates coagulation and gradually tilts the balance towards chronic DIC.

Works of Bick et al ${ }^{[2]}$ and Saxena et al ${ }^{[3]}$ laid down the criteria on chronic DIC and our present study also confirms that chronic DIC occurs in lung cancers of epithelial origin especially in advanced stages.

\section{Materials And Methods}

The following study was conducted in the department of Pathology (Haematology section) of IPGMER, Kolkata, in the period June 2002 to May 2003 in collaboration with Department of Radiotherapy and Chest medicine. Permission was taken from IEC. Finance of the study was provided by authority (Approx. Rs. 10500).

For the study case were selected from Department of Radiology and Chest Medicine after diagnosis but before treatment of malignancy and then blood samples are taken from patients and following tests were done. 
1) Complete haemogram; 2) Prothrombin time (P.T); 3) Activated partial thromboplastin time (A.P.T.T); 4) Fibrinogen degradation products estimation by FDP D` Dimer assay by a semi quantitative method.

Total number of lung cancer patient in the study was 35 . Controls were also 35 , selected from healthy staffs and students of the medical college. Criteria for control were 1) non-smokers2) healthy and active3) no clinically proven disease of lung and liver, heart and haematopoetic system.

All technical parts of procedures are done as mentioned in Dacie, Lewis (Practical Haematology). ${ }^{[4]} 5 \mathrm{cc}$ of blood was drawn from each patient. For estimation of P.T \& A.PT.T, $1.8 \mathrm{cc}$ of blood was taken in a test tube containing $0.2 \mathrm{ml}$ of citrate. Rest $3 \mathrm{ml}$ blood was taken in EDTA vial. Two smears were made on glass slides by two drops of EDTA blood and stained by Wright's stain and estimated for differential count of WBC, approximate total count of WBC which is also estimated by COULTER counter and Platelet count is done from stained blood smear and whenever necessary platelet count is done in Neubauer chamber using ammonium oxalate as diluents in usual method. Haemoglobin is estimated using cyanmethaemoglobin method with the help of Drabkin solution and colorimeter. P.T and A.PT.T-Platelet poor plasma is prepared from citrated blood (blood: citrate $=9: 1$ ).

P.T. -Measures the clotting time of plasma in presence of thromboplastin and overall screens the extrinsic and common pathway. Commercially available rabbit brain extracts calibrated against international reference thromboplastin; water bath; and stopwatch is used.

A.P.T.T-Measures the clotting time of plasma after the activation of contact factor but without added tissue thromboplastin and so screens the intrinsic pathway. Kaolin; commercial phospholipid; $0.025 \mathrm{~mol} / \mathrm{L} \mathrm{Ca}$ $\mathrm{Cl} 2$ are used. Paired results are taken with normal plasma taken as control and mean result is given in both PT \& A.P.T.T.

FDP D`Dimer assay-XLFDP reagent (polystyrene latex particle, coated with mouse monoclonal Anti

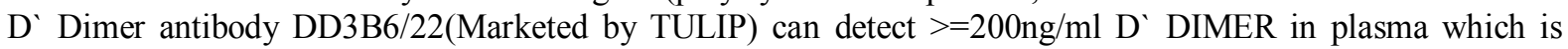
taken as positive. Positive \&Negative controls, phosphate buffer diluents for semi quantitative assay and serial diluting plate, all are supplied with the kit. It is a semi quantitative assay. Plasma is serially diluted as 1:2, 1:4, 1:8 and the like and the highest dilution for agglutination with reagent antibody are noted. Time for each agglutination reaction is fixed at 3 minutes as per the literature with the kit. D' Dimer level=200xd where $\mathrm{d}=$ highest dilution of plasma for agglutination.

\section{Results}

It is evident that passive smoking must have a strong role in lung cancer of females and responsibility of their husband cannot be denied. (Table 2).

From Table 3 it is evident that most cases belong to (51-60years) age group amounting to $40 \%$ and followed by (61-70 years) age group amounting to $28.56 \%$.

Table 5 shows squamous cell carcinoma is the preponderant carcinoma in our country like India probably due to universality of smoking. Due to lack of health consciousness only stage III \& IV cancers are available in our study. Only cancers of epithelial origin -sq. cell carcinoma, adenocarcinoma, bronchoalveolar carcinoma are taken for study.

It is evident from Table 6 that Platelet count is high in 18(51.43\%) patients and normal in 17(48.57\%) as in review article "haemostatic changes in malignancy- a review" by Saxena $\mathrm{R}$ et al ${ }^{[3]}$ it is seen that malignancy chronic DIC shows normal to high platelet count. Platelet count at the range of 1.5-4.5 lakhs/cubic $\mathrm{mm}$ was taken as normal range. Above 4.5 lakhs as high and below1.5 lakhs as low were considered.

Present study confirms the findings of Saxena $R^{[3]}$ and text book like Wintrobe's haematology ${ }^{[6]}$ that APTT in chronic DIC is more or less normal. (Normal range 30-40 seconds) (Table 7).

Table 8 shows that in present study normal PT in most of the cases which is also in favour of chronic DIC. (normal range 11-16 seconds). chronic DIC.

Present study shows in 19(54.28\%) cases FDP D’ Dimer is increased. (Table 9) It is surest marker of

Table 10 depicts that this study shows elevated D'Dimer level in $20 \%$ of stage- III and $60 \%$ in stageIV consistent with the finding of Chopra et al (2001) ${ }^{[7]}$ in which he observed chronic DIC in $31.25 \%$ cases of stage-III and $61.53 \%$ of stage-IV epithelial lung cancers. D Dimer` level above $200 \mathrm{ng} / \mathrm{ml}$ is taken as positive or high as per literature in the kit.

This study shows $85.71 \%$ normal haemoglobin and decreased haemoglobin in $14.29 \%$ cases. This has nothing to do with DIC as because decreased haemopoesis can occur with metastasis, circulating tumor product like TNF-alpha and they are sufficient to cause anemia in any type of malignancy ( $10 \mathrm{gm} \%$ was taken as cut off point of normal haemoglobin for female and $12 \mathrm{gm} \%$ for that of male) (Table 11).

As per Table 12 present study shows rise in TLC and neutrophilia as a result of inflammation and stimulation of bone marrow by products released by tumor cells. TLC more than 11000/cubic mm was taken as high count (as per Dacie Lewis) ${ }^{[4]}$. Percentage of neutrophil $>80 \%$ was taken as high DC of neutrophil. 
Table 13 shows that in present study, out of 19 cases with raised FDP D` Dimer 11 cases that means $57.9 \%$ have high platelet count and out of total 35 studied cases 18 cases $(51.43 \%)$ have high platelet count (vide Table 6). PT \& APTT are not significantly raised and normal in most of the cases. So in majority of cases platelet count is raised. (Patients with raised D' Dimers showing any change in other coagulation parameters) 19 cases with increased FDP s are considered $100 \%$.

\section{DISCUSSION}

As per Wintrobes "Clinical Haematology" ${ }^{[6]}$ in contrast to inherited coagulation disorder acquired coagulation defects are multi factorial. Because of the compound nature of the haemostatic defect as in carcinomas the severity of bleeding correlates poorly with the results of the laboratory tests.

DIC can occur in-1) Carcinomas of lung, pancreas, breast etc; 2) Metastatic carcinoids, neuroblstomas, rhabdomyosarcomas; 3) Malignancies of haematopoetic system.

Mechanisms by which DIC is initiated- a) platelet adhesion\& aggregation; b) activated extrinsic \& intrinsic path. These exceed the normal compensatory process. In cancers tumor emboli and released tumor vesicles are thought to enter circulation and act as thromboplastin. These factors interacts with factor VII and activate the extrinsic pathway of coagulation. Tumor cell surface expression of tissue factors had been demonstrated (DovrakHF,Vandewalter et al). ${ }^{[8]}$ Results of quantitative studies demonstrate accelerated turnover of platelets, fibrinogen but plasma level of prothrombin which is consumed severely in vitro experiments is often normal in DIC especially in chronic DIC.

Intravascular fibrin formation- formation of fibrin in the form of small strands and micro clots is the immediate result of DIC. 1) Fibrinolysis- common finding in DIC; 2) Many of the thromboplastic substances released from the tumor mass also contain Plasminogen activators. Finally factor XIIa activates plasminogen by inter acting with normal proactivators and its kin in the system. Fibrinogen degradation product (FDP s)- the above mentioned processes degrade fibrinogen proteolytically.

Biologic effects of these products are - a) Acts as anti thrombin; b) Inhibit polymerization of fibrin; c) Produce structurally defective polymers; d) Impair platelet function; e) Impair RE system clearance function; f) Smaller peptides of 3000-4000 D inhibit smooth muscle activity.

Pathophysiology of chronic compensated DIC is same as acute but results from weak intermittently activated stimulus .In such cases destruction and production of coagulation factors are evenly balanced. Clinical features of chronic DIC- Recurrent episodes of epistaxis, extensive echymosis, migratory thrombophlebitis etc. are seen. It can be converted to acute DIC with dramatic suddenness following surgical trauma or pulmonary embolism. So chronic DIC is an intermediate step between acute DIC and hypercoagulable state. Some neurological symptoms may occur in chronic DIC.

A newly coined term is used by Joist et al [9] AICF (ACCELERATED INTRAVASCULAR COAGULATION \& FIBRINOLYSIS).

In view of shortage of resources platelet count, PT, APTT, complete haemogram and FDP'D Dimer assay was taken as minimum criteria for assessment of DIC in this study.

Essential for treatment of low grade or chronic DIC is the treatment of underlying cause.

1) Supportive therapy is a must; 2) If thromboembolism continues anticoagulant therapy is indicated but aggressive anticoagulant therapy is contraindicated; 3) Therapy must be individualized depending on age site, stage of cancer severity of haemorrhage, infection and chemotherapy; 4) Surgery and radiotherapy may tilt the balance towards acute DIC and trigger severe bleeding and thrombosis.

Considering the above mentioned modalities proper study of incidence of chronic DIC in epithelial lung cancers is that much important.

\section{TABLES}

Table 1: Sex ratio among cases

\begin{tabular}{|l|l|l|}
\hline Sex & No of cases & Percentage of total cases \\
\hline Male & 27 & $77.1 \%$ \\
\hline Female & 8 & $22.9 \%$ \\
\hline Total & 35 & $100 \%$ \\
\hline
\end{tabular}

Table 2: Distribution of cases according to smoking habit.

\begin{tabular}{|l|l|l|l|l|l|l|l|l|l|}
\hline Sex & $\begin{array}{l}\text { Total } \\
\text { case }\end{array}$ & Smoker & $\%$ & Non Smoker & $\%$ & $\begin{array}{l}\text { Other spouse } \\
\text { (smoker) }\end{array}$ & $\%$ & Other spouse (non smoker) & $\%$ \\
\hline Male & 27 & 26 & 96.3 & 1 & 3.7 & 0 & 0 & 27 & 100 \\
\hline Female & 8 & 0 & 0 & 8 & 100 & 8 & 100 & 0 & 0 \\
\hline
\end{tabular}


Table 3: Age distribution of cases

\begin{tabular}{|l|l|l|}
\hline Range of age & No. of cases & percentage \\
\hline $31-40$ years & 3 & $8.57 \%$ \\
\hline $41-50$ years & 7 & $20 \%$ \\
\hline $51-60$ years & 14 & $40 \%$ \\
\hline $61-70$ years & 10 & $28.56 \%$ \\
\hline Above 70 years & 1 & $2.87 \%$ \\
\hline Total & 35 & $100 \%$ \\
\hline
\end{tabular}

Table 4: Distribution of cases as per stage of cancer

\begin{tabular}{|l|l|l|}
\hline Stage of cancer & No of cases & Percentage \\
\hline I & NIL & $0 \%$ \\
\hline II & NIL & $0 \%$ \\
\hline III & 5 & $14.28 \%$ \\
\hline IV & 30 & $85.72 \%$ \\
\hline Total & 35 & $100 \%$ \\
\hline
\end{tabular}

Procedures of staging was taken from Harrison`s Principles of Internal Medicine as per Criteria laid down ${ }^{[5]}$

Table 5: Distribution of cases according to type of malignancy

\begin{tabular}{|cl|l|l|}
\hline \multicolumn{2}{|c|}{ Type of malignancy } & No of cases & percentage \\
\hline 1$)$ & Adenocarcinoma & 7 & $20 \%$ \\
\hline 2$)$ & Squamous cell carcinoma & 27 & $77.13 \%$ \\
\hline 3$)$ & Bronchoalveolar carcinoma & 1 & $2.87 \%$ \\
\hline
\end{tabular}

Table 6: Categorization of patients according to platelet count

\begin{tabular}{|l|l|l|}
\hline Platelet count & No of cases & percentage \\
\hline High range & 18 & $51.43 \%$ \\
\hline Normal range & 17 & $48.57 \%$ \\
\hline Low range & NIL & $0 \%$ \\
\hline Total & 35 & $100 \%$ \\
\hline
\end{tabular}

Table 7: APTT status in chronic DIC.

\begin{tabular}{|l|l|l|}
\hline A.P.T.T & No of cases & Percentage \\
\hline High & 4 & $11.11 \%$ \\
\hline Normal & 31 & $88.89 \%$ \\
\hline Low & NIL & $0 \%$ \\
\hline Total & 35 & $100 \%$ \\
\hline
\end{tabular}

Table 8: PT status in chronic DIC.

\begin{tabular}{|l|l|l|}
\hline PT & No of cases & Percentage \\
\hline High & 2 & $5.75 \%$ \\
\hline Normal & 33 & $94.3 \%$ \\
\hline Low & NIL & $0 \%$ \\
\hline Total & 35 & $100 \%$ \\
\hline
\end{tabular}

Table 9: FDP D’ Dimer status in chronic DIC

\begin{tabular}{|l|l|l|}
\hline FDP D`Dimer & No of cases & Percentage \\
\hline High & 19 & $54.28 \%$ \\
\hline Normal & 16 & $45.72 \%$ \\
\hline Low & NIL & $0 \%$ \\
\hline Total & 35 & $100 \%$ \\
\hline
\end{tabular}

Table 10: Distribution of cases as per high or normal FDP D'Dimer

\begin{tabular}{|l|l|l|l|l|l|l|}
\hline Stages & cases & High FDP D` Dimer & $\begin{array}{l}\text { Percentage of high FDP } \\
\text { D`Dimer }\end{array}$ & $\begin{array}{l}\text { Normal D` } \\
\text { Dimer }\end{array}$ & $\begin{array}{l}\text { Percentage of } \\
\text { normal }\end{array}$ \\
\hline III & 5 & 1 & $20 \%$ & 4 & $80 \%$ \\
\hline IV & 30 & 18 & $60 \%$ & 12 & $40 \%$ \\
\hline
\end{tabular}

Table 11: Haemoglobin status of patients

\begin{tabular}{|l|l|l|}
\hline Haemoglobin & No of cases & Percentage \\
\hline Normal & 30 & $85.71 \%$ \\
\hline Decreased & 5 & $14.29 \%$ \\
\hline Total & 35 & $100 \%$ \\
\hline
\end{tabular}


Table 12: Neutrophil count \& total count of WBC in patients

\begin{tabular}{|l|l|l|l|l|}
\hline Range & Neutrophil in DC & Percentage & Total count of WBC & Percentage \\
\hline High & 28 cases & $80 \%$ & 28 cases & $80 \%$ \\
\hline Normal & 7 cases & $20 \%$ & 7 cases & $20 \%$ \\
\hline Low & NIL & $0 \%$ & NIL & $0 \%$ \\
\hline Total & 35 & $100 \%$ & 35 & $100 \%$ \\
\hline
\end{tabular}

Table 13: PT, APTT \& platelet count status in patients with raised FDP D` Dimer.

\begin{tabular}{|l|l|l|l|l|l|l|l|}
\hline Range & PT & $\%$ & APTT & $\%$ & Platelet count & $\%$ & FDP D`Dimer \\
\hline & No of cases & & No of cases & & No of cases & & No of cases \\
\hline Raised & 2 & $10.53 \%$ & 4 & $21 \%$ & 11 & $57.9 \%$ & 19 \\
\hline Normal & 17 & $89.47 \%$ & 15 & $79 \%$ & 8 & $42.1 \%$ & 0 \\
\hline Total & 19 & $100 \%$ & 19 & $100 \%$ & 19 & $100 \%$ & 19 \\
\hline
\end{tabular}

Table 14: Different coagulation parameters in control group

\begin{tabular}{|l|l|l|l|l|l|l|l|l|}
\hline Range & Platelet count & $\%$ & APTT & $\%$ & PT & $\%$ & FDP D Dimer & $\%$ \\
\hline High & NIL & $\%$ & NIL & $\%$ & NIL & $\%$ & NIL & $\%$ \\
\hline Normal & 35 & $100 \%$ & 35 & $100 \%$ & 35 & $100 \%$ & 35 & $100 \%$ \\
\hline Low & NIL & $0 \%$ & NIL & $0 \%$ & NIL & $0 \%$ & NIL & $0 \%$ \\
\hline Total & 35 & & & & & & \\
\hline
\end{tabular}

\section{Conclusion}

From various investigation and studies it is evident that one of the most common acquired coagulopathy in lung cancers of epithelial origin is chronic DIC. For this study 35cases of epithelial lung cancers (proven by bronchoscopy, radiology, cytology and/ biopsy) before onset of anticancer therapy were chosen. Complete haemogram, PT, APTT, FDP D’Dimer assay were taken as methods. In our study PT was normal in 33 i.e94.3\% cases. APTT was normal in31i.e.88.89\% cases. FDP D' Dimer assay which was taken as the main criteria was found to be mild to moderately raised signifying chronic DIC in 19 i.e. $54.28 \%$ cases. These 19 cases with chronic DIC had normal PT in $89.47 \%$ (17cases) and normal APTT in $79 \%$ (15 cases) and elevated platelet count in $57.9 \%$ (11 cases). These criteria fit exactly with criteria laid down by R Saxena et al ${ }^{[3]}$ and Bick et al ${ }^{[2]}$.

Thus at the end it can be safely concluded that chronic DIC is very common in lung cancer of epithelial origin. A larger cohort and further investigation can be more fruitful in calculating and categorizing the incidences.

This types of studies will further help to individualize lung cancers of epithelial origin on the basis of DIC and will help to form a treatment protocol to prevent bleeding and/ coagulopathic catastrophes in them.

\section{References}

[1] Mersky.C,JohnsonA.J,Kleiner G.J and Wohl. H.The defibrination syndrome:Clinical features and Laboratory diagnosis.British journal of haematolgy, 1967;13:528-548

[2] Bick RL, DIC current concepts of etiology, pathology and diagnosis. J Clinical Oncol, 1987, Dec; 5(12)

[3] Saxena R, Tyagi S, Haematologic changes in malignancies-A review.:Indian journal of Haematology and blood transfusion 2001; vol 19 no-2:39-40

[4] Dacie.John V, Lewis, S M, Practical Haematology, (10th edition,Churchil-Livingstone Publication)2010:41-54,398-401

[5] Harrison's Principle of Internal Medicine, editor-Longo et al (McGraw-Hills Publication-18 ${ }^{\text {th }}$ edition 2012) vol-1:745-747

[6] Wintrobe's Clinical Haematology, editor- John P. Greer et al (Wolters Kluwer/ Lippincot Williams and Wilkin`s, 12 ${ }^{\text {th }}$ edition), 2009, vol-2:1439-1441

[7] Chopra R,Sing I et al. Incidence of chronic DIC in epithelial malignancies.Indian journal of Haematology and blood transfusion ,2001,vol 19 no-4:92-93

[8] Dovrak H.F et al. Procoagulant activity associated with plasma membrane vesicles shed by cultured tumor cells Can Res, 1983; 62:14-31

[9] Joist, J. Heinrich. "AICF and DIC in liver cirrhosis: expressions of a hypercoagulable state." The American journal of gastroenterology 94.10 (1999): 2801-2803. 